\title{
Effects of a kappa distribution function of electrons on incoherent scatter spectra
}

\author{
S. Saito ${ }^{1}$, F. R. E. Forme $^{2}$, S. C. Buchert ${ }^{1}$, S. Nozawa ${ }^{1}$, R. Fujii ${ }^{1}$ \\ ${ }^{1}$ Solar-Terrestrial Environment Laboratory, Nagoya University, Furo-cho, Chikusa-ku, Nagoya, 464-8601, Japan \\ ${ }^{2}$ CETP-Universite Versailles St-Quentin en Yvelines, 10-12, Av. de l'Europe, 78140 Velizy, France
}

Received: 21 January 2000 / Revised: 31 May 2000 / Accepted: 22 June 2000

\begin{abstract}
In usual incoherent scatter data analysis, the plasma distribution function is assumed to be Maxwellian. In space plasmas, however, distribution functions with a high energy tail which can be well modeled by a generalized Lorentzian distribution function with spectral index kappa (kappa distribution) have been observed. We have theoretically calculated incoherent scatter spectra for a plasma that consists of electrons with kappa distribution function and ions with Maxwellian neglecting the effects of the magnetic field and collisions. The ion line spectra have a double-humped shape similar to those from a Maxwellian plasma. The electron temperatures are underestimated, however, by up to $40 \%$ when interpreted assuming Maxwellian distribution. Ion temperatures and electron densities are affected little. Accordingly, actual electron temperatures might be underestimated when an energy input maintaining a high energy tail exists. We have also calculated plasma lines with the kappa distribution function. They are enhanced in total strength, and the peak frequencies appear to be slightly shifted to the transmitter frequency compared to the peak frequencies for a Maxwellian distribution. The damping rate depends on the electron temperature. For lower electron temperatures, plasma lines for electrons with a $\kappa$ distribution function are more strongly damped than for a Maxwellian distribution. For higher electron temperatures, however, they have a relatively sharp peak.
\end{abstract}

Key words: Ionosphere (auroral ionosphere; plasma waves and instabilities) - Space plasma physics (kinetic and MHD theory)

Correspondence to: S. Saito

e-mail: susaito@stelab.nagoya-u.ac.jp

\section{Introduction}

Incoherent scattering is a very powerful tool to investigate plasma in the Earth's upper atmosphere and in the laboratory. Using incoherent scatter (IS) spectra, one can derive various parameters such as the density, the ion and electron temperature, the ion velocity, and others. It is well known that the velocity distribution functions of ions and electrons in a plasma are Maxwellian, if the ions and the electrons are in thermal equilibrium. Often, however, external energy input causes significant deviations of the distribution functions from a Maxwellian. For example, in the Earth's ionosphere a high electric field in combination with ionneutral interaction leads to a bi-Maxwellian or even a toroidal distribution function of the ions (St.-Maurice and Schunk, 1979). The corresponding incoherent scatter (IS) ion line was explored in detail (Raman et al., 1981). Zheng et al. (1997) have calculated the spectrum of another type of non-Maxwellian electron distribution function, the super-Gaussian distribution $\left(f(v) \propto \exp \left(-\left(v / v_{t h}\right)^{x}\right), x>2\right)$. In this work we investigate the effects of a kappa distribution function of electrons on the IS spectrum.

Theoretically it has been shown that the velocity distribution function obeys a power-law at energies higher than critical energy when a superthermal radiation field is present (Hasegawa et al., 1985). Such a power-law functional dependence is an approximation to the more general distribution, the generalized Lorentzian or kappa distribution (Baumjohann and Treumann, 1996). Measurements of electron energy spectra with space craft have been successfully modeled with kappa distributions (Vasyliunas, 1968; Christon et al., 1988; Bryant, 1999). Also plasma turbulence can interact with both thermal and suprathermal particles and generate suprathermal tails of the particle velocity distribution (Galeev, 1989). For plasmas in which both electrons and ions have kappa distribution, Summers and Thorne (1991) have calculated the modified plasma 
dispersion function theoretically, and found general properties of ion acoustic waves and Langmuir waves (see also Summers et al., 1996). Since incoherent scatter spectra are closely related to those waves, some qualitative expectation of the spectra can be obtained, although it is actually assumed that only electrons have kappa distribution while ion distribution function remains Maxwellian.

An IS spectrum consists of two characteristic features, the ion line and the plasma line. The ion line is an echo that corresponds to ion acoustic waves found in the low frequency range (for a transmitter frequency of several hundred $\mathrm{MHz}$, in the range of a few $\mathrm{kHz}$ ). Since the ion line contains information about ions as well as electrons, it is the ion line that is used to derive the ionospheric parameters. Therefore, it is important to know the effects of the kappa distribution function on the ion line for the correct derivation of the parameters. This knowledge would be very important, for example, for estimating the momentum balance of the ionosphere or the threshold of plasma instabilities. The other characteristic feature is that plasma lines are received in the high frequency range. This feature corresponds to Langmuir waves found around $\left(\omega_{p e} / 2 \pi\right)\left(1+3 k^{2} \lambda_{D}^{2}\right)^{\frac{1}{2}}$, where $\lambda_{D}$ is the electron Debye length. Usually the lines appear at several $\mathrm{MHz}$ up- and downshifted from the transmitter frequency depending on electron density and temperature. Since ions are too heavy to oscillate at the high frequency range, the contribution of ions to Langmuir waves is negligible. The shape and position of the plasma lines are determined by electrons (and also by the radar $\mathbf{k}$-vector). Therefore, it is expected that a kappa distribution of electrons has clearer effects on the plasma line than on the ion line. This may be useful in obtaining a correct analysis of the ion line by providing more information about electrons.

In the Earth's topside ionosphere the European Incoherent Scatter (EISCAT) radars observe frequently enhanced electron temperatures caused by soft precipitation which is also associated with ion outflow (Wahlund et al., 1992; Ogawa et al., 1999). The data analysis is usually carried out under the assumption of Maxwellian distribution functions. However, under the conditions of particle precipitation and plasma waves, instabilities can be triggered and the turbulent electric field enhances velocity-space diffusion and distorts the electron distribution function. Therefore, one cannot exclude the possibility that the electron distribution develops a high energy tail which should be modeled by a kappa distribution. In this work we calculate the IS ion and plasma lines for a plasma with electron kappa distributions. The effects and possible misinterpretations of a Maxwellian analysis are investigated.

In Sect. 2 the theoretical basis for calculating the IS spectra is presented. In Sect. 3 the ion line is discussed, and the errors caused by a Maxwellian analysis are examined in Sect. 4. Section 5 investigates the plasma lines resulting from an electron kappa distribution.

\section{Theory}

The procedure of the theoretical calculation of an IS spectrum is described by Sheffield (1975). Scattered power in the frequency range $\mathrm{d} \omega$ and in the solid angle $\mathrm{d} \Omega$ with wave vector $\mathbf{k}$ is given by

$P_{s} \mathrm{~d} \Omega \mathrm{d} \omega \propto n_{e 0} S(\mathbf{k}, \omega)$

where $n_{e 0}$ is the electron density. $S(\mathbf{k}, \omega)$ is the spectral density function given by

$S(\mathbf{k}, \omega)=\lim _{\gamma \rightarrow 0} \frac{2 \gamma}{V}\left\langle\frac{\left|n_{e}(\mathbf{k}, \omega-i \gamma)\right|^{2}}{n_{e 0}}\right\rangle$,

where $n_{e}(\mathbf{k}, \omega-i \gamma)$ is the Fourier- in space and Laplacein time transform of the electron density $n_{e}(\mathbf{r}, t)$,

$$
\begin{aligned}
& n_{e}(\mathbf{k}, \omega-i \gamma) \\
& \quad=\int_{-\infty}^{+\infty} \mathrm{e}^{i \mathbf{k} \cdot \mathbf{r}} \mathrm{d} \mathbf{r} \int_{-\infty}^{+\infty} n_{e}(\mathbf{r}, t) \mathrm{e}^{-i(\omega-i \gamma)} \mathrm{d} t \quad(\gamma>0) .
\end{aligned}
$$

When no magnetic field exists, or $\mathbf{k}$ is parallel to the magnetic field, the spectral density function is given by

$S(\mathbf{k}, \omega)=2\left|\frac{\left(1+C_{i}\right)}{\epsilon}\right|^{2} B_{e}+2 Z\left|\frac{C_{e}}{\epsilon}\right|^{2} B_{i}$

where $Z$ is the charge number of ions. Functions $C_{e, i}$, $B_{e, i}$, and $\epsilon$ in Eq. (4) are defined taking into account the effect of collisions through a BGK model as follows:

$$
\begin{aligned}
C_{j} & =\frac{1}{1+D_{j}} \int_{-\infty}^{+\infty} \mathrm{d} v \frac{q_{j}^{2} n_{j 0}}{m_{j} \epsilon_{0} k^{2}} \frac{\mathbf{k} \cdot \partial f_{j 0}(v) / \partial v}{\omega-\mathbf{k} \cdot v-i v_{j}} \\
B_{j} & =\frac{v_{j}}{\left|1+D_{j}\right|^{2}} \int_{-\infty}^{+\infty} \mathrm{d} v \frac{f_{j 0}(v)}{(\omega-\mathbf{k} \cdot v)^{2}+v_{j}^{2}}-\frac{\left|D_{j}\right|^{2}}{v_{j}\left|1+D_{j}\right|^{2}}
\end{aligned}
$$

where

$D_{j}=i v_{j} \int_{-\infty}^{+\infty} \mathrm{d} v \frac{f_{j 0}(v)}{\omega-\mathbf{k} \cdot v-i v_{j}}$.

$v_{j}$ is the collision frequency, $f_{j 0}(v)$ the distribution function, $m_{j}$ the mass, $q_{j}$ the electric charge, $n_{j 0}$ the density, $\epsilon_{0}$ the electric permittivity of the vacuum, and $\omega$ the angular frequency. Here $j$ can be $e$ or $i$ to denote electrons or ions, respectively. $\epsilon\left(\mathbf{k}, \omega, v_{e}, v_{i}\right)$ is the plasma dielectric function,

$\epsilon\left(\mathbf{k}, \omega, v_{e}, v_{i}\right)=1+C_{i}+C_{e}$.

In integrating Eqs. (5) and (6), the integrals of a form need to be calculated.

$$
\int_{-\infty}^{+\infty} \mathrm{d} v \frac{f_{j 0}(v)}{\omega-\mathbf{k} \cdot v-i v_{j}}
$$

The integrand has a pole at $\mathbf{k} \cdot v=\omega-i v_{j}$. The integration path must be above the pole because we have 
defined the Laplace transform with negative imaginary part of frequency and taken the limit of $\gamma \rightarrow 0$ even in the case of a collisionless plasma.

Equations (4)-(8) are valid for arbitrary distribution functions. It is well known that the particle distribution function becomes Maxwellian when the particles are in thermal equilibrium. Therefore, in usual incoherent scatter analysis one can assume that $f_{j 0}$ is Maxwellian,

$f_{M}(v)=\frac{1}{\left(\sqrt{\pi} v_{t h, M}\right)^{3}} \exp \left(-\frac{v^{2}}{v_{t h, M}^{2}}\right)$

where $v_{t h, M}$ is the characteristic velocity (or thermal velocity) of the Maxwellian distribution function given by

$v_{t h, M}=\sqrt{\frac{2 k_{B} T}{m}}$

where $k_{B}$ is the Boltzman constant, $T$ the temperature, and $m$ the mass. Later the temperature of different distribution functions is discussed. Therefore, the temperature must be clearly defined in a consistent way for any distribution functions. We define the temperature along a certain direction by the second order moment of a one-dimensional distribution function $f^{(1)}\left(v_{\|}\right)$, or mean kinetic energy of particles,

$$
\left\langle\frac{1}{2} m v_{\|}^{2}\right\rangle=\int_{-\infty}^{+\infty} \frac{1}{2} m v^{2} f\left(v_{\|}\right) \mathrm{d} v \equiv \frac{1}{2} k_{B} T .
$$

In fact, Eq. (11) has been derived using this definition.

However, as mentioned in Sect. 1, distribution functions with high energy tail have been often observed in space plasmas when energy input maintaining the high energy tail exists. It is known that such distribution functions can be well modeled by a generalized Lorentzian function with spectral index $\kappa$ (kappa distribution). The one-dimensional kappa distribution function is given by

$f_{\kappa}^{(1)}(v)=\frac{1}{\sqrt{\pi} v_{t h, \kappa}} \frac{\Gamma(\kappa+1)}{\kappa^{3 / 2} \Gamma(\kappa-1 / 2)}\left(1+\frac{v^{2}}{\kappa v_{t h, \kappa}^{2}}\right)^{-\kappa}$

where $v_{t h, \kappa}$ is also the characteristic velocity (or thermal velocity) of the kappa distribution function given by

$v_{t h, \kappa}=\sqrt{\frac{2 \kappa-3}{\kappa} \frac{k_{B} T}{m}}$

which satisfies the relation (12) when $\kappa>3 / 2$. (Summers and Thorne, 1991) Kappa distribution functions for different values of $\kappa$ are compared with Maxwellian in Fig. 1. It should be noted that the smaller the index $\kappa$ is, the more high energy electrons exist. In the limit of $\kappa \rightarrow \infty$, the kappa distribution converges to Maxwellian.

To evaluate the spectral density function, it is assumed that the ion distribution function is Maxwellian because it is unlikely to be influenced severely by plasma waves or turbulence. Following Sheffield (1975), Eqs. (5)-(7) for ions become as follows:

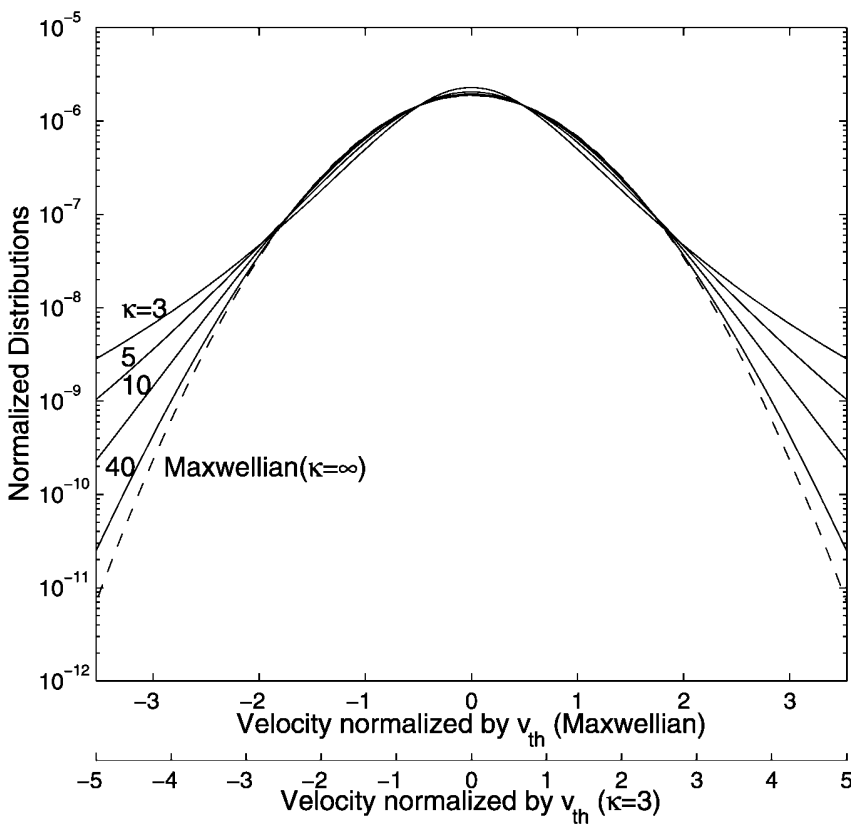

Fig. 1. A comparison between Maxwellian and kappa distribution with $\kappa=3,5,10$, and 40. Maxwellian distribution (dashed line) corresponds to $\kappa=\infty$. x-axes are indicated by velocities normalized by $v_{t h, M}$ and $v_{t h, \kappa=3}$

$$
\begin{aligned}
C_{i} & =\frac{1}{1+D_{i}} \frac{1}{Z} \frac{2 \omega_{p i}^{2}}{k^{2} v_{t h, M}^{2}}\left[1+\xi_{M} Z_{-}\left(\xi_{M}\right)\right] \\
D_{i} & =-\frac{i v_{i}}{k v_{t h, M}} Z_{-}\left(\xi_{M}\right) \\
B_{i} & =-\frac{1}{k v_{t h, M}\left|1+D_{i}\right|^{2}} \operatorname{Im}\left[Z_{-}\left(\xi_{M}\right)\right]-\frac{\left|D_{i}\right|^{2}}{v_{i}\left|1+D_{i}\right|^{2}}
\end{aligned}
$$

where

$$
\begin{aligned}
Z_{-}\left(\xi_{M}\right) & =\frac{1}{\sqrt{\pi}} \int_{-\infty}^{+\infty} \frac{\mathrm{e}^{-\xi_{M}^{2}}}{s-\xi_{M}} \mathrm{~d} s \\
& =i \sqrt{\pi} \exp \left(-\xi_{M}^{2}\right)\left(\operatorname{erf}\left(i \xi_{M}\right)-1\right)
\end{aligned}
$$

and

$\xi_{M}=\frac{\omega-i v_{i}}{k v_{t h, M}}$.

'erf' is the complex error function. Here the ion plasma frequency defined is introduced by

$\omega_{p i}=\left(\frac{n_{i 0} Z^{2} e^{2}}{m_{i} \epsilon_{0}}\right)^{\frac{1}{2}}$.

The function $Z_{-}\left(\xi_{M}\right)$ given by Eq. (18) is very similar to the plasma dispersion function (Fried and Conte, 1961) except for the sign of the second term of the right hand side. When the electron distribution function along the line-of-sight is a generalized Lorentzian distribution (kappa distribution), i.e. $\int_{-\infty}^{+\infty} \mathrm{d} v_{\perp} f(v)=f_{\kappa}^{(1)}\left(v_{\|}\right)$, where $v_{\perp}$ and $v_{\|}$are the velocities perpendicular to and parallel to the line-of-sight, respectively, we can write 


$$
\begin{aligned}
C_{e}= & \frac{1}{1+D_{e}} \frac{2 \omega_{p e}^{2}}{k^{2} v_{t h, \kappa}^{2}}\left[1-\frac{1}{2 \kappa}+\xi_{\kappa} Z_{\kappa,-}^{*}\left(\xi_{\kappa}\right)\right] \\
D_{e}= & -\frac{i v_{e}}{k v_{t h, \kappa}}\left(\frac{\kappa}{\kappa-3 / 2}\right)\left(\frac{\kappa-1}{\kappa}\right)^{3 / 2} \\
& \times Z_{\kappa-1,-}^{*}\left(\sqrt{\frac{\kappa-1}{\kappa}} \xi_{\kappa}\right) \\
B_{e}= & -\frac{1}{k v_{t h, \kappa}\left|1+D_{e}\right|^{2}} \operatorname{Im}\left[\left(\frac{\kappa}{\kappa-3 / 2}\right)\left(\frac{\kappa-1}{\kappa}\right)^{3 / 2}\right. \\
& \left.\times Z_{\kappa-1,-}^{*}\left(\sqrt{\frac{\kappa-1}{\kappa}} \xi_{\kappa}\right)\right]-\frac{\left|D_{e}\right|^{2}}{v_{e}\left|1+D_{e}\right|^{2}}
\end{aligned}
$$

where

$$
Z_{\kappa,-}^{*}\left(\xi_{\kappa}\right)=\frac{\kappa^{\kappa-1 / 2} \Gamma(\kappa+1)}{\sqrt{\pi} \Gamma(\kappa-1 / 2)} \int_{-\infty}^{+\infty} \frac{\mathrm{d} s}{\left(s-\xi_{\kappa}\right)\left(s^{2}+\kappa\right)^{\kappa+1}}
$$

and

$\xi_{\kappa}=\frac{\omega-i v_{e}}{k v_{t h, \kappa}}$.

$Z_{\kappa,-}^{*}\left(\xi_{\kappa}\right)$ can be analytically expressed for positive integer $\kappa$ as

$$
\begin{aligned}
Z_{\kappa,-}^{*}\left(\xi_{\kappa}\right)= & -\frac{\kappa-1 / 2}{2 \kappa^{3 / 2}} \frac{\kappa !}{(2 \kappa) !} \sum_{l=0}^{\kappa} \frac{(\kappa+l) !}{l !}(-i)^{\kappa-l} \\
& \times\left(\frac{2}{\left(\xi_{\kappa} / \sqrt{\kappa}\right)-i}\right)^{\kappa+1-l}
\end{aligned}
$$

Our $Z_{\kappa,-}^{*}$ is the complex conjugate of the modified dispersion function $Z_{\kappa}^{*}$ introduced by Summers and Thorne (1991) because we use a different complex frequency definition in the Laplace transform.

\section{Incoherent scatter spectra with kappa distribution I: ion lines}

We have calculated IS spectra for a kappa distribution plasma for different values of $\kappa$ and compared them with those from a Maxwellian plasma with the same set of parameters, $n_{e}, T_{i}$, and $T_{e}$. In our calculation, the following assumptions were made.

1. Electron distribution function along the magnetic field line is a kappa distribution and that in the plane perpendicular to the line-of-sight is Maxwellian, while that of the ions (in all directions) remains Maxwellian because of their heavy mass.

2. Effects of the magnetic field are not taken into account. This assumption should be justified when calculating the IS spectra from a field-aligned measurement.

3. The probing radio wave frequency is $933 \mathrm{MHz}$ (wave number $k_{0}=19.55 \mathrm{~m}^{-1}$ ) corresponding to the Troms UHF radar, one of the EISCAT radar systems. Assuming backscattering (the transmitting and re- ceiving site are the same), this means that the wave number $k$ becomes $39.1 \mathrm{~m}^{-1}\left(=2 k_{0}\right)$.

4. Although we can take the effects of collisions into account, we set $v_{i, e}=0$ for simplicity since we are interested in phenomena in the $F$ region where the collisions are too rare to deform an IS spectrum noticeably.

5. In the case of the $F$ region, we assume that the dominant ion is $\mathrm{O}^{+}\left(m_{i}=16 \mathrm{amu}\right)$.

6. The mean drift velocity of ions, $v_{i}$, is zero.

Figure 2 shows an example of our calculations of the IS spectra for various $\kappa$ at the low frequency range which corresponds to the ion acoustic fluctuations (ion lines). The spectra show double-humped shapes similar to the case of Maxwellian plasma. For the same set of $n_{e}, T_{i}$, and $T_{e}$, the total scattered power becomes stronger, the spectral width remains almost the same, the spectral peaks are slightly downshifted, and the depth of the valley between two spectral peaks becomes shallower. With increasing $\kappa$, the spectrum converges to a Maxwellian spectrum. Indeed, the IS spectra for a kappa distribution plasma are quite different from those for a Maxwellian plasma, although ion distribution function which mainly contributes to the ion lines is assumed to remain Maxwellian.

The increase in the total scattered power can be understood in the following way. The level of excitation of the ion line is, among other functions, a function of the local electron density for velocities around the phase velocity of the corresponding ion acoustic waves. For the chosen set of parameters, $v_{t h, M} \sim 3 \cdot 10^{5} \mathrm{~m} \mathrm{~s}^{-1}$, the

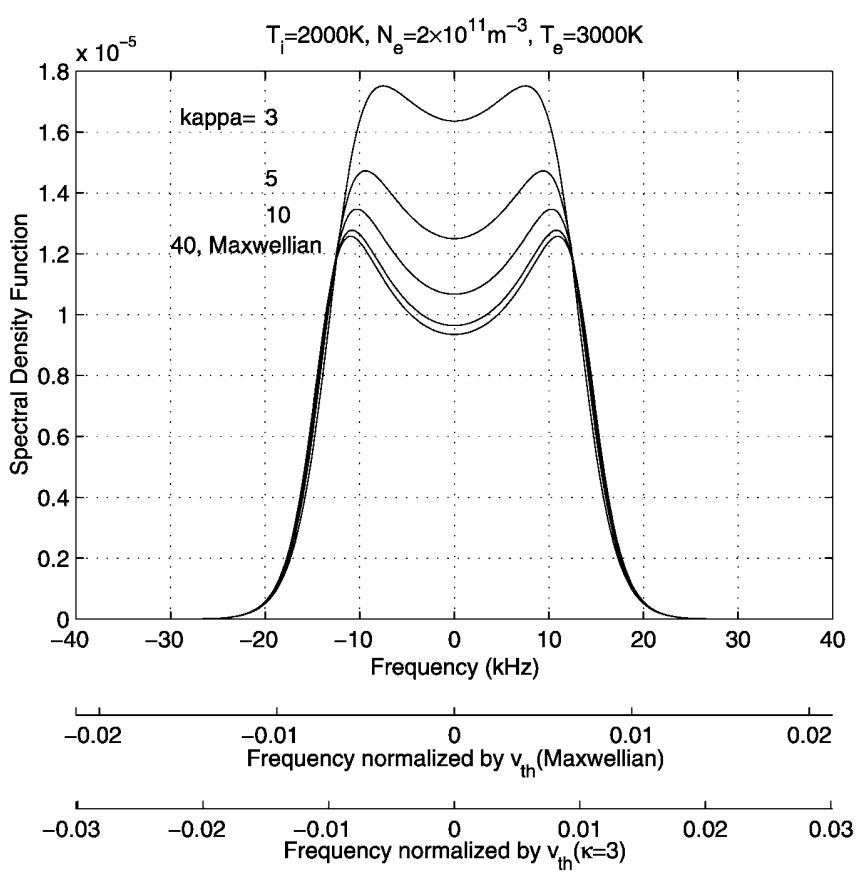

Fig. 2. IS spectral density functions of kappa distribution plasma for a different spectral index, $\kappa=3,5,10$, and 40, together with one from Maxwellian plasma $(\kappa=\infty)$ are shown. For this case, $n_{e}=2 \cdot 10^{11} \mathrm{~m}^{-3}, T_{i}=2000 \mathrm{~K}, T_{e}=3000 \mathrm{~K}$, and $m_{i}=16$ a.m.u. $x$ axes are indicated by corresponding velocities normalized by $v_{t h, M}$ and $v_{t h, \kappa=3}$ as well as by a frequency in $\mathrm{kHz}$ 
ion acoustic waves at frequencies around $\pm 10 \mathrm{kHz}$ correspond to $v / v_{t h, M} \sim \pm 0.0053$. Looking at Fig. 1, it is easy to see that in this velocity range the kappa distribution function has higher values than the Maxwellian distribution. Therefore, the excitation level of the ion line is higher.

To explain the differences of the IS spectra from the viewpoint of thermal fluctuations in a plasma more quantitatively, we have numerically solved the following dispersion equation (Thorne and Summers, 1991),

$$
\begin{gathered}
1+\frac{2 \omega_{p e}^{2}}{k^{2} v_{t h, e}}\left[\left(1-\frac{1}{2 \kappa}\right) \frac{\omega}{k v_{t h, e}} Z_{\kappa}^{*}\left(\frac{\omega}{k v_{t h, e}}\right)\right] \\
+\frac{2 \omega_{p i}^{2}}{k^{2} v_{t h, i}}\left[1-\frac{\omega}{k v_{t h, i}} Z\left(\frac{\omega}{k v_{t h, i}}\right)\right]=0
\end{gathered}
$$

where $\omega$ is a complex frequency, $Z$ and $Z_{\kappa}^{*}$ are the plasma dispersion function (Fried and Conte, 1961) and the
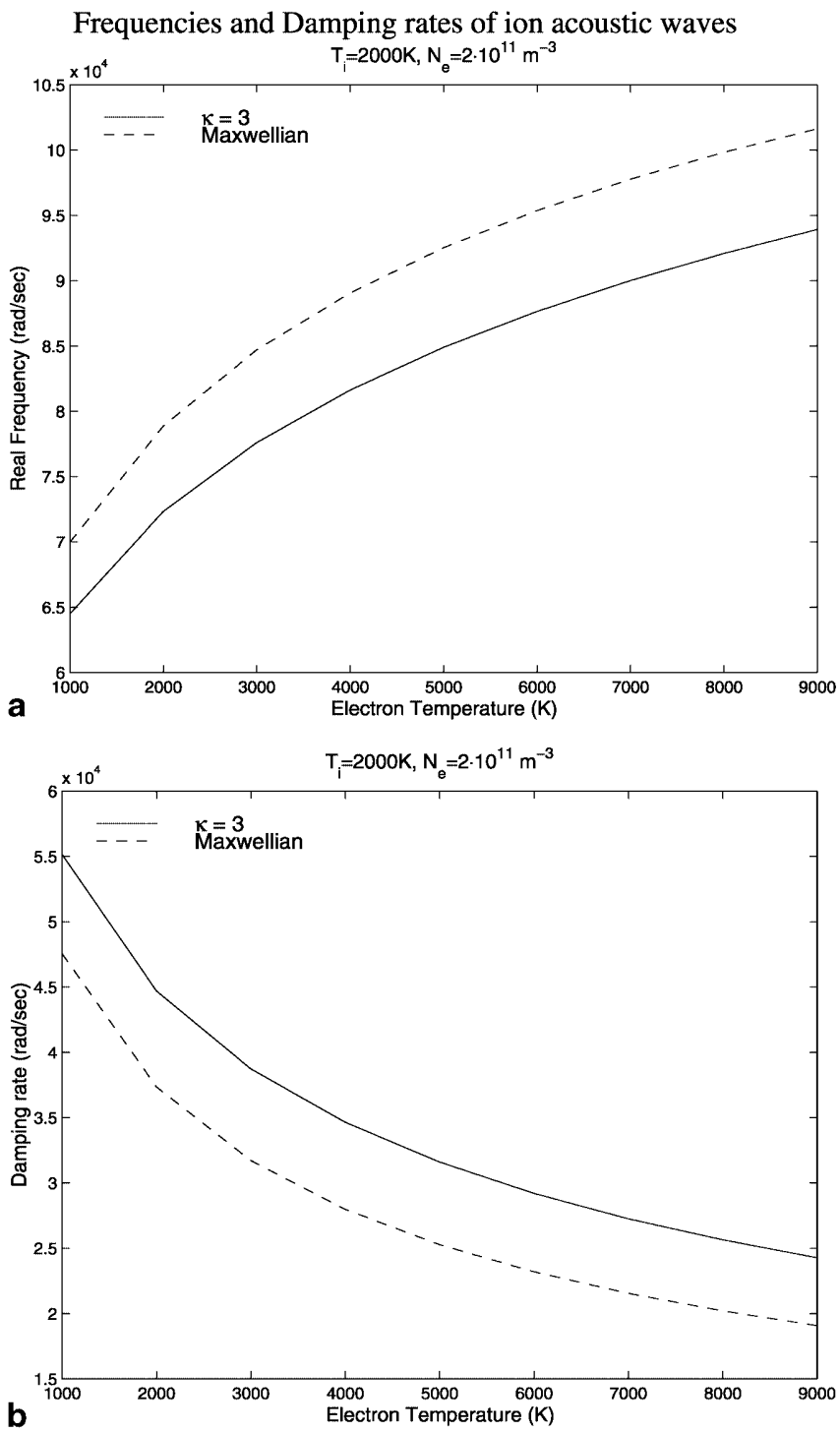

Fig. 3a, b. Solutions of dispersion equation for ion acoustic waves for $\kappa=3$ and Maxwellian. a Frequency and $\mathbf{b}$ damping rate are plotted over electron temperature. Ion temperature and electron density are fixed to $1000 \mathrm{~K}$ and $n_{e}=2 \cdot 10^{11} \mathrm{~m}^{-3}$, respectively modified plasma dispersion function (Summers and Thorne, 1991), respectively. For ion acoustic waves, we have obtained the results represented in Fig. 3. It can be seen that the frequencies of the ion acoustic waves for $\kappa=3$ are downshifted compared to the Maxwellian case. This corresponds to our result (Fig. 2) that the spectral peaks are slightly downshifted for kappa distribution. The damping rates of the ion acoustic waves for kappa distribution are stronger than those for Maxwellian. This also corresponds to the fact that the valley between the two spectral peaks becomes shallower.

If the spectra from a kappa distribution plasma are interpreted by assuming a Maxwellian plasma, the derived parameters would be quite different. So it is worth knowing quantitatively how much the differences of interpreted plasma parameters, such as $n_{e}, T_{i}$, and $T_{e}$, could be by using Maxwellian spectra in fitting. In the next section, we will estimate how large the differences could become as a result of assuming a Maxwellian distribution function if the actual distribution is a kappa distribution.

\section{Difference of plasma parameters as a result of fitting with Maxwellian distribution}

In our analysis, IS spectra for a kappa distribution plasma with parameters $\left(n_{e}, T_{i}, T_{e}\right.$, and $\left.\kappa\right)$, are fitted by an IS spectra model for a Maxwellian plasma using the least square method. $n_{e}, T_{i}$, and $T_{e}$ derived by the standard analysis are determined. Figure 4 is an example of fitting a Maxwellian spectra to $n_{e} S(\mathbf{k}, \omega)$ calculated according to the procedure in the previous section. For this case, the ion temperature and electron density are almost the same, but the electron temperature is significantly underestimated to only $1800 \mathrm{~K}$ instead of $3000 \mathrm{~K}$. The

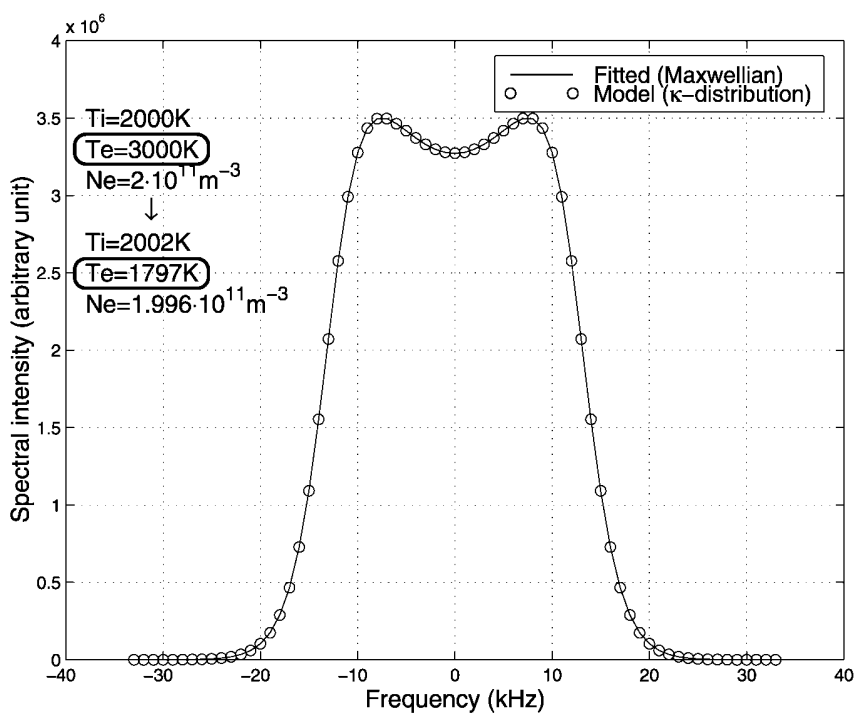

Fig. 4. An example of fitting on an IS spectrum $\left(n_{e} S(\mathbf{k}, \omega)\right)$ with kappa distribution by an IS spectrum with Maxwellian distribution. The model spectrum (plotted by circles) assumes $T_{i}=2000 \mathrm{~K}$, $T_{e}=3000 \mathrm{~K}, n_{e}=2 \cdot 10^{11} \mathrm{~m}^{-3}$, and $\kappa=3$. Solid line shows fitted IS spectrum with Maxwellian distribution function with $T_{i}=2002 \mathrm{~K}$, $T_{e}=1797 \mathrm{~K}, n_{e}=1.996 \cdot 10^{11} \mathrm{~m}^{-3}$ 
results for various $\kappa$ with the same plasma parameters as in Fig. 4 are shown in Fig. 5. Again $n_{e}$ and $T_{i}$ change little, but the fitted $T_{e}$ is always lower than assumed for the kappa distribution spectrum. The underestimation is up to $40 \%$ when $\kappa$ is very small (more deviation from Maxwellian). The results with different sets of $n_{e}, T_{i}, T_{e}$, and $\kappa$ are similar (not shown here).

It is surprising that the estimated electron density remains almost the same even though the total scattered power is larger for the spectrum with the kappa distribution. This can be understood by considering the relationship between the total scattered power and the ionospheric parameters. For the Maxwellian distribution, the total scattered power $P_{s}$ is proportional to $N_{e} /\left(1+T_{e} / T_{i}\right)$. Therefore, the estimated $N_{e}$ is proportional to $P_{s}\left(1+T_{e} / T_{i}\right)$. By lowering the estimation of $T_{e}$, the estimated $N_{e}$ remains almost the same even though $P_{s}$ for the kappa distribution spectrum is larger than that for the Maxwellian for the same set of $N_{e}, T_{i}$, and $T_{e}$.

This underestimate of $T_{e}$ may also be surprising, but this result is rather reasonable. Ion lines are sensitive to the derivative of the ion and electron distribution functions at the velocity range corresponding to the phase velocity of ion acoustic waves. For electrons, this velocity range is the very narrow region of the center of
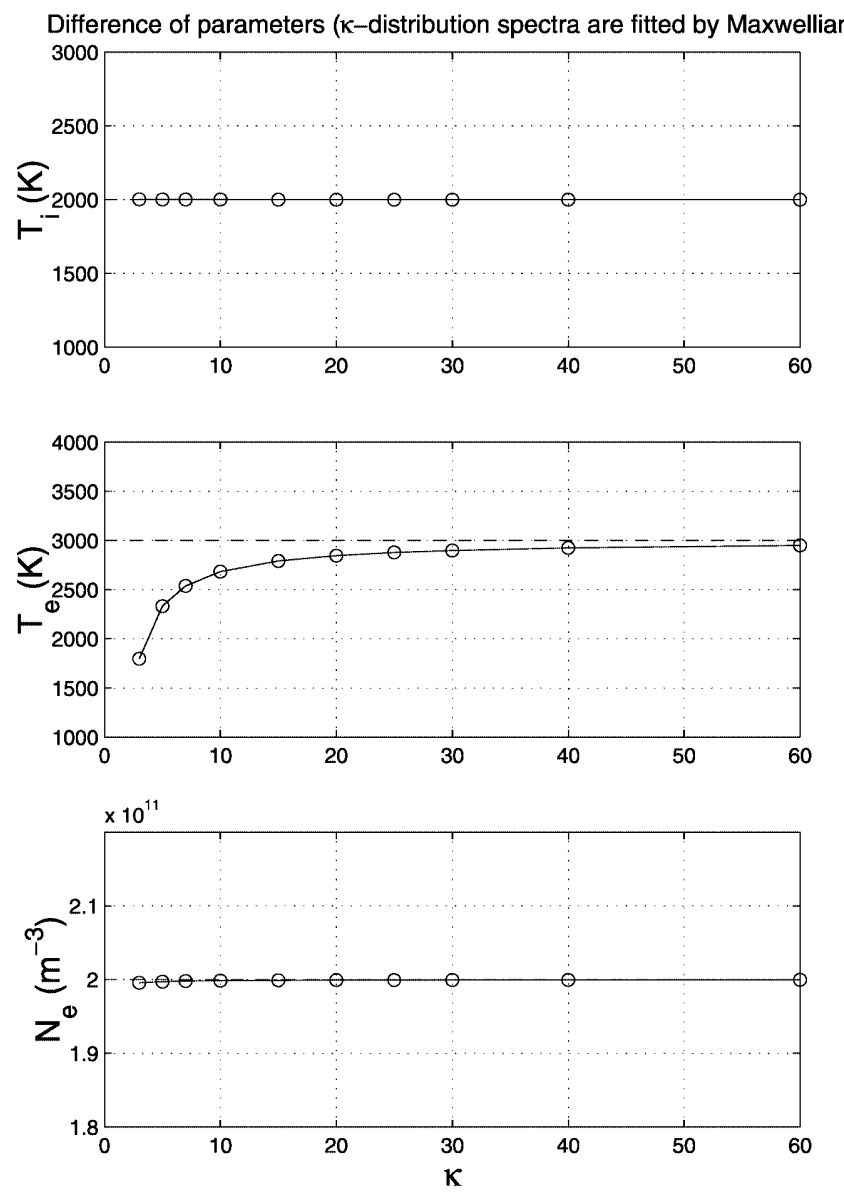

Fig. 5. The difference of parameters when kappa distribution IS spectra are interpreted by assuming Maxwellian distribution for $T_{i}, T_{e}$, and $n_{e}$ are shown from the top to the bottom. They are plotted as relative errors to real (kappa distribution) parameters their distribution function as shown in Fig. 1. There, the width of a kappa distribution function is narrower than that of a Maxwellian with the same temperature, and a similar width of a Maxwellian distribution function for a lower temperature.

The same analysis was performed for the EISCAT VHF radar (transmitted frequency $224 \mathrm{MHz}$ ) and the EISCAT Svalbard radar $(500 \mathrm{MHz})$. The same results were obtained for both the radars (not shown here) as the EISCAT UHF radar shown already.

\section{Incoherent scatter spectra with kappa distribution II: plasma lines}

The kappa distribution contains many more particles in the high energy tail. That should make a significant effect in the high frequency part of the IS spectrum. Bjørnå and Trulsen (1986) have calculated the effect of a photo electron flux whose energy spectrum are modeled by power law on plasma lines. They find that the total cross section at the EISCAT UHF radar frequency is increased but the position remains the same as it is in the Maxwellian case. Summers and Thorne (1991) have shown that Langmuir waves of a kappa distribution plasma are more damped than those of Maxwellian plasma, and that the frequency of the Langmuir waves are closer to $\omega_{p e}$. Hence when $k \lambda_{D} \ll 1$ it is expected that plasma lines show stronger damping and have a smaller shift in frequency.

We have calculated plasma lines for various $T_{e}$ cases with fixed $\kappa, T_{i}$ and $N_{e}$ and compared them to Maxwellian lines as shown in Fig. 6. It can be seen that plasma lines for $\kappa$ distribution are more strongly damped than they are for Maxwellian for lower $T_{e}(\lesssim 4000 \mathrm{~K})$. For higher $T_{e}$ cases $(\gtrsim 6000 \mathrm{~K})$, however, plasma lines for Maxwellian are more strongly damped than they are for $\kappa$ distribution. For $T_{e}=10000 \mathrm{~K}$, a plasma line for Maxwellian becomes very flat while that for $\kappa$ distribution is still relatively sharp. At these high electron temperatures, $k \lambda_{D}$ becomes comparable to unity. Thus according to Thorne and Summers (1991) wave damping for Maxwellian exceeds that for $\kappa$ distribution. Our results for higher temperatures are still consistent with previous works.

In Fig. 7 a calculation of plasma lines for a specific set of $T_{e}$ and $n_{e}$ and for various values of $\kappa$ is shown and compared with a Maxwellian calculation. The positions are slightly shifted toward the transmitter frequency. This becomes clearer for smaller $\kappa$.

This smaller Doppler frequency of the plasma line with smaller $\kappa$ can be explained in the following way. The Langmuir dispersion relation is more exactly $\omega \simeq \omega_{p e}\left(1+3 k^{2}\left\langle v^{2}\right\rangle / \omega_{p e}^{2}\right)^{1 / 2}$, where $\left\langle v^{2}\right\rangle$ is the mean square velocity of electrons which is the second order moment of the distribution function. Since $\left\langle v^{2}\right\rangle=\frac{2 \kappa-3}{2 \kappa} \frac{k_{B} T}{m}$ for the kappa distribution, $\left\langle v^{2}\right\rangle$ is smaller for the smaller $\kappa$, and consequently the frequency of the Langmuir wave becomes smaller. This means that the Doppler frequency of the plasma line should be smaller for the smaller $\kappa$. 

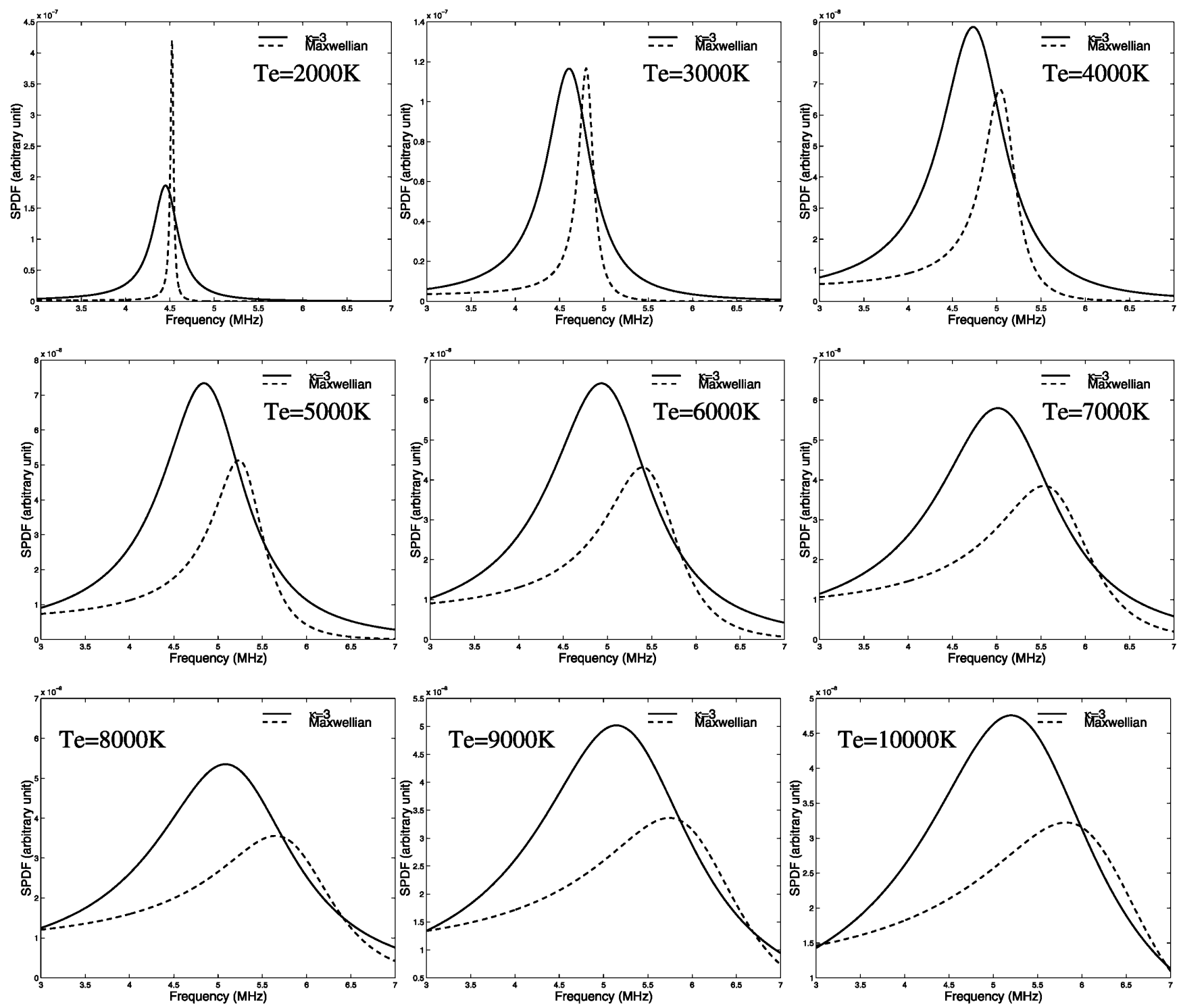

Fig. 6. Plasma lines (upshifted) for various electron temperatures (2000-10 $000 \mathrm{~K}$ ) for $\kappa=3$ (solid line) and Maxwellian (dashed line) are shown. Electron density is fixed to $2 \cdot 10^{11} \mathrm{~m}^{-3}$. x-axis ranges are all 3-7 $\mathrm{MHz}$. But $y$-axis ranges are arbitrary for each plot

\section{Conclusion}

We have derived incoherent scatter spectra for a plasma when the velocity distribution is a generalized Lorentzian or kappa distribution function.

We have calculated incoherent scatter spectra for a plasma that consists of electrons with a kappa distribution function and ions with a Maxwellian, and compared the spectra with those for a Maxwellian plasma. The ion lines have a double-humped shape similar to those for a Maxwellian plasma. The electron temperatures are, however, underestimated by up to $40 \%$ when interpreting them assuming Maxwellian distributions for both ions and electrons. Ion temperatures and electron densities are less affected.

This kind of analysis could be used when soft electron precipitation is inferred or when intense wave activity is observed. For example, electron temperature enhancements caused by soft electron precipitation which is also associated with ion outflow are often observed with EISCAT radars. Our results suggest that in such cases electron temperatures derived with the assumption of a Maxwellian distribution function might be underestimated. This can lead to the incorrect estimation of forces like ambipolar electric field through the electron temperature. Our analysis could be extended to fit the spectral index $\kappa$ together with other parameters, although it would require high signal-tonoise ratio. The correctly analyzed data would significantly contribute to the understanding of ionospheric phenomena such as the ion upflow and enhanced ion acoustic echoes.

Another application of our analysis is for a heating experiment in which the ionosphere is artificially modified and heated by powerful electromagnetic waves. In such an experiment, one would expect a strongly distorted electron distribution function. Although it is not certain if the electron distribution function can be 


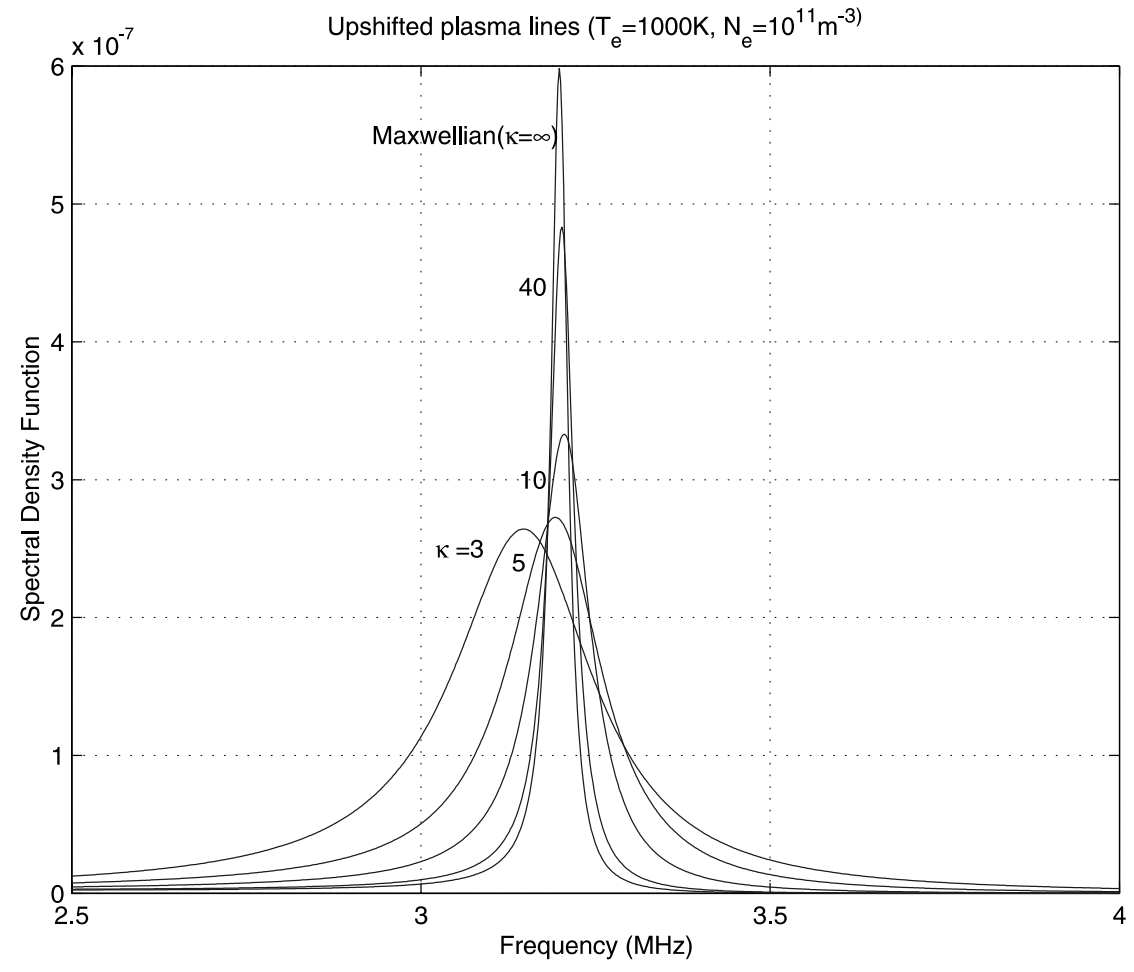

Fig. 7. Plasma lines (upshifted) are plotted for a different spectral index, $\kappa=3,5,10$, and 40 , together with plasma line of Maxwellian plasma $(\kappa=\infty)$ modeled by the kappa distribution, we believe that it is a good example to test our analysis.

We have also calculated the plasma lines for the kappa distribution function for electrons. We have found a significant difference in the shape and a slight difference in the frequency shift. Although practically it is still difficult to measure the shape of a plasma line as we have calculated, simultaneous measurements of plasma lines with ion lines would provide more precise information on the electron distribution function.

Acknowledgement. We are indebted to the director and staff of EISCAT for operating the facility and supplying the data. EISCAT is an international association supported by Finland (SA), France (CNRS), the Federal Republic of Germany (MPG), Japan (NIPR), Norway (NFR), Sweden (NFR), and the United Kingdom (PPARC). This study was supported in part by a Grant-in-aid for International Scientific Research (09044074) and by Grants-in-aid for Scientific Research A (08304030) and B (08454135, 11440144), and C (11640441) by the Ministry of Education, Science, Sports and Culture, Japan.

Topical Editor M. Lester thanks P. Guio and another referee for their help in evaluating this paper.

\section{References}

Baumjohann, W., and R. A. Treumann, Basic space plasma physics, Imperial College Press, London, 1996.

Bjørnå, N., and J. Trulsen, Effect of a power low particle flux on the ionospheric incoherent scattering cross section, Physica Scripta, 33, 284-288, 1986.

Bryant, D., Electron acceleration in the aurora and beyond, Institute of Physics Publishing, Bristol and Philadelphia, 75, 1999.

Christon, S. P., D. G. Mitchell, D. J. Williams, L. A. Frank, C. Y. Huang, and T. E. Eastman, Energy spectra of plasma sheet ions and electrons from $\sim 50 \mathrm{eV} / e$ to $\sim 1 \mathrm{MeV}$ during plasma temperature transitions, J. Geophys. Res., 93, 2562-2572, 1988.
Fried, B. D., and S. D. Conte, The plasma dispersion function, Academic Press, New York, 1961.

Galeev, A. A., Spectra of plasma turbulence, particle acceleration and heating by plasma waves in the interacting plasma, in Plasma waves and instabilities at comets and in magnetospheres, AGU, Washington DC, 1989.

Hasegawa, A., M. Kunioki, and M. Duong-van, Plasma distribution function in a superthermal radiation field, Phys. Rev. Lett., 54, 2608-2610, 1985.

Ogawa, Y., R. Fujii, S. C. Buchert, S. Nozawa, S. Watanabe, and A. P. Van Eyken, Simultaneous EISCAT Svalvard and VHF radar observations of ion upflows at different aspect angles, Geophys. Res. Lett., 1999 (in press).

Raman, R. S. V., J. P. St.-Maurice, and R. S. B. Ong, Incoherent scattering of radar waves in the auroral ionosphere, J. Geophys. Res., 86, 4751-4762, 1981.

Sheffield, J., Plasma scattering of electromagnetic radiation, Academic Press, New York, 1975.

St.-Maurice, J.-P., and R. W. Schunk, Ion velocity distributions in the high latitude ionosphere, Rev. Geophys. Space Phys., 17, 99134, 1979.

Summers, D., and R. M. Thorne, The modified plasma dispersion function, Phys. Fluids, B3, 1835-1847, 1991.

Summers, D., R. M. Thorne, and H. Matsumoto, Evaluation of the modified plasma dispersion function for half-integral indices, Phys. Plasmas, 3, 2496-2501, 1996.

Thorne, R. M., and D. Summers, Landau damping in space plasmas, Phys. Fluids, B3, 2117-2123, 1991.

Vasyliunas, V. M., A survey of low-energy electrons in the evening sector of the magnetosphere with OGO 1 and OGO 3, J. Geophys. Res., 73, 2839-2885, 1968.

Wahlund, J.-E., F. R. E. Forme, H. J. Opgenoorth, M. A. L. Persson, E. V. Mishin, and A. S. Volokitin, Scattering of electromagnetic waves from a plasma: enhanced ion acoustic fluctuations due to ion-ion two-stream instabilities, Geophys. Res. Lett., 19, 1919-1922, 1992.

Zheng, J., C. X. Yu, and Z. J. Zheng, Effects of nonMaxwellian (super-Gaussian) electron distribution on the spectrum of Thomson scattering, Phys. Plasmas, 4, 27362740, 1997. 